\title{
Treatment strategies in intermediate cervical neoplasia: Implications of radical surgery
}

\author{
K.W.M. VAN DELFT and H.J.M.M. MERTENS \\ Department of Gynecology, Orbis Medical Center, 6162 BG Sittard-Geleen, The Netherlands
}

Received November 5, 2010; Accepted March 16, 2011

DOI: $10.3892 / \mathrm{ol} .2011 .289$

\begin{abstract}
Treatment strategies for cervical intraepithelial neoplase (CIN)2 lesions differ among gynaecologists. To evaluate the differences in management of treatment with subsequent implications, all surgical treatment strategies and follow-up methods were retrospectively analysed for patients with intermediate dysplasia of the cervix. This study aimed to evaluate expectant management strategies and the effect of biopsy prior to radical surgery in CIN2. Patients diagnosed with a CIN2 lesion at the Orbis Medical Center in The Netherlands between 2006 and 2007 were retrospectively analysed. The follow-up ended on 1st January 2009. All 141 patients with CIN2 lesions were included; 109 had no previous history of any CIN lesion. Of the 109 patients, 12\% ( $n=13)$ underwent an immediate radical surgical excision of the transformation zone (LLETZ procedure) and $85 \%(n=93)$ underwent a local biopsy. After the lesion was biopsied, expectant management was selected for $59 \%$ of the patients. Subsequent smears were normal in $40 \%$ of the patients. Of the patients with abnormal smears in follow-up, the LLETZ procedure was performed in $86 \%$ of the patients $(n=25)$. Of these cases, persistent disease was observed in $14 \%$ of the patients. After an immediate LLETZ procedure without prior biopsy, follow-up smears were abnormal in $31 \%$ of the patients. Persistent disease was significantly lower following radical excision of the lesion with a diagnostic biopsy versus without one (14 versus $31 \%$ ). After expectant management, the rate of persistent disease was $53 \%(\mathrm{p}<0.001)$. Overall, the rate of persistent disease was $7 \%$. Due to the high rate of persistent disease and the lower rate of overtreatment, CIN2 lesions should be treated by the excisional procedure. To restrict persistent disease, a biopsy is recommended prior to the actual treatment, since a higher rate of abnormal smears was observed in the follow-up after immediate radical excisions in the first visit.
\end{abstract}

Correspondence to: Dr H.J.M.M. Mertens, Orbis Medical Center, Gynecology Department, 6162 BG Sittard-Geleen, The Netherlands E-mail: h.mertens@orbisconcern.nl

Key words: dysplasia, cervical intraepithelial neoplasia2, LLETZ

\section{Introduction}

Cervical intraepithelial neoplasia (CIN), as a possible precancerous disease, is predominantly observed in females of a reproductive age (1). A total of $70 \%$ of all cervical dysplasias are detected by population screening with cytology $(2,3)$. The incidence of cervical cancer in The Netherlands is 9 per 100,000 women (approximately 700 patients) $(2,4)$. In The Netherlands, cervical population screening is available from the age of 30 years, at 5-year intervals. Due to standardised cervical cancer screening programs, the prevalence of cervical cancer has declined (1).

Treatment strategies for CIN2 differ among gynaecologists in The Netherlands. Some of them prefer no surgical intervention and expectant management, whereas others prefer to perform radical excisions of the transformation zone. The histological diagnosis of CIN2 is extremely heterogeneous and has high inter-observer variation. Epidemiologic and molecular data suggest that CIN2 is a diverse group with some cases similar to CIN3 and some similar to productive HPV infection.

Of routinely screened women in the United States, 5\% were found to have an abnormal cervical smear (5). The overall incidence of CIN was 2.7 per 1,000 females (5). Per 1,000 females, CIN1 was found in 1.2; CIN2 in 0.8 and CIN3 in 0.7 females (5). The highest rate of CIN1 (5.1 per 1,000; $95 \%$ CI 2.2-9.2) was observed in women between 20 and 24 years of age (5). The highest rates of CIN2 (3.8 per 1,000; 95\% CI 1.9-6.3) and CIN3 (4.1 per 1,000; 95\% CI 2.1-6.7) were observed in females between 25 and 29 years of age (5).

Some cervical dysplasia may be a self-limiting disease. Of all CIN1 lesions, regression is observed in $60 \%$ of cases and development to cervical carcinoma is observed in less than $1 \%$ $(1,6-8)$. For CIN2 lesions, regression and malignant development is noted in 40 and $5 \%$ of cases $(1,6-8)$; for CIN3 lesions, regression and malignant development is noted in 31 and $22 \%$ of cases, respectively $(1,6-9)$. This possibly underestimates the true invasive potential of CIN3, since even a small diagnostic biopsy may be curative for some female individuals (9).

Inappropriate management of CIN lesions may increase the risk of cervical cancer and/or result in overtreatment of non-progressing and even self-limiting CIN lesions (1). Due to the high rate of persisting dysplasia and the possibility of progression to invasive cancer, excisional procedures should be performed $(1,6-8)$. 
Due to their heterogeneous pathology, CIN1, CIN2 and CIN3 may be combined for treatment decisions. Follow-up studies have shown that despite marginal relative differences in behaviour, the three lesions are more likely to persist or progress than to regress (1). CIN2 and CIN3 lesions share a number of biological characteristics usually associated with true cervical cancer precursors (1). There is widespread agreement that treatment of CIN2 and CIN3 reduces both incidence of and mortality from invasive cervical cancer (1). However, options for the conservative management of adolescents with CIN2 and CIN3 have been expanded (1).

Few prospective studies focusing purely on CIN2 management are currently available. This analysis delineates the natural history of CIN2 and the effects of surgical treatments for CIN2. This study aimed to evaluate expectant management strategies and the effect of biopsy prior to radical surgery for CIN2.

\section{Materials and methods}

Patients presenting with CIN2 lesions at the Orbis Medical Center in Sittard between 2006 and 2007 were retrospectively analysed. The patients underwent at least one colposcopic and histologic examination during this period of time.

Cytologic and histologic examinations were followed up until 1st January 2009. In this medical center, two pathologists examine cervical lesions.

The average and median age of the 141 patients with CIN2 lesions during the study period was 39 years (18-84 years). According to the pathologist, the lesions were situated in the ectocervical epithelium in $97.6 \%(\mathrm{n}=120)$, in the endocervical epithelium in $0.8 \%(\mathrm{n}=1)$ and in both epithelial types in $1.6 \%$ $(n=2)$ of cases.

Previous cytologic examinations were normal in $3 \%$ of patients and abnormal in 89\% (PAP2 in 8\%; PAP3A in 70\%; PAP3B in $8 \%$ and PAP4 in 3\%). No cytologic examination was previously performed in $8 \%$ of cases. In these cases, the CIN2 lesions were detected after portio amputation $(n=2)$ or after other prior histological abnormalities in either a therapeutic or a diagnostic program $(\mathrm{n}=9)$. Patient history showed that $23 \%(n=32)$ of patients underwent cervical biopsy(-ies) or cervical surgery.

A Mann-Whitney U-test was performed on the data. $\mathrm{P}<005$ was considered to be statistically significant.

\section{Results}

Of the 109 patients (77\%) who had never had prior cervical interventions, a diagnostic biopsy was performed on $85 \%$ of patients $(n=93)$ and an immediate radical excision of the transformation zone was performed on $12 \%$ of patients $(n=13)$. In $3 \%$ of all patients $(n=3)$, CIN2 lesions were accidentally found after a prolapse operation. Previous cytologic examinations in the biopsy group were normal in 2.2\% ( $\mathrm{n}=2)$ and abnormal in $97.8 \%$ [PAP2 in $4.2 \%(\mathrm{n}=4), \mathrm{PAP} 3 \mathrm{~A}$ in $81.7 \%$ $(n=76)$, PAP3B in $7.5 \%(n=7)$ and PAP4 in $4.3 \%(n=4)]$. Previous cytologic examinations in the immediate LLETZ group were all abnormal [PAP2 in $23.1 \%(\mathrm{n}=4)$, PAP3A in $69.2 \%(\mathrm{n}=9)$ and PAP3B in $7.7 \%(\mathrm{n}=1)]$. The decision to perform either a LLETZ procedure or a diagnostic biopsy was made, depending on the PAP smear and on the decision of the gynaecologist based on the colposcopy exam.

Post-biopsy. After the lesion was biopsied (pathological result CIN2), expectant management was selected for $59 \%$ of patients $(n=55)$. One of the reasons for expectant management is a histological result which is concordant with the cytological result. Subsequent smears were normal in $40 \%(\mathrm{n}=22)$ and abnormal in $53 \%(n=29)$ of patients, with $7 \%$ of patients $(n=4)$ being lost to follow-up. Of the patients with abnormal smears in the follow-up $(n=29)$, radical surgical excisions of the transformation zone were performed using the LLETZ procedure in $86 \%(\mathrm{n}=25)$; local biopsies were performed on $7 \%(\mathrm{n}=2)$ of patients and exconisations on $7 \%(\mathrm{n}=2)$ of patients. The results showed no dysplasia in $8 \%(n=2)$, CIN1 in $15 \%(n=4)$, CIN2 in $56 \%(n=14)$ and CIN3 in 20\% ( $n=5)$ of patients.

After the lesion was biopsied, LLETZ procedures were performed in a second visit in $31 \%(n=29)$ of patients. In $86 \%$ $(n=25)$ of patients, follow-up smears were normal. In $14 \%$ $(n=4)$ of patients, the smears were abnormal; thus other local biopsies $(\mathrm{n}=3)$ or a hysterectomy $(\mathrm{n}=1)$ were performed.

An exconisation was performed on $4 \%$ of the patients. In 3 out of 4 patients, follow-up smears were normal, whereas in 1 case the follow-up smear was abnormal (PAP3A).

Post-immediate LLETZ. After immediate radical excision of the transformation zone (LLETZ) without a prior biopsy, follow-up smears were abnormal in $31 \%$ of patients. Therefore, persistent disease was significantly lower after radical excision of the lesion with a prior diagnostic biopsy versus without (14 versus $31 \%$ ). Following expectant management, the rate of persistent disease was $53 \%(\mathrm{p}<0.001)$ (Fig. 1).

Overall, the rate of persistent disease was $7.3 \%$. The average and median times between abnormal cytological and histological results were 37 and 32 days (range 12-224), respectively. The average and median times between a diagnostic biopsy and subsequent treatments were 113 and 56 days (range 9-428), respectively. The mean time between the first visit for the biopsy and the second for the LLETZ procedure was 36 days.

\section{Discussion}

For CIN2 lesions, regression and malignant development is observed in 40 and $5 \%$ of cases, respectively $(1,6-8)$. The interindividual variation in the management of individuals with a CIN2 lesion is high among different (oncologic) gynaecologists. Some prefer expectant management, others perform radical excisions.

The population of this cohort is older than in most series of cervical premalignant diseases. The population in The Netherlands is initially invited for the national screening program at the age of 30 . In many countries, cervical screening starts at a younger age. Difference in age may therefore explain the higher average and median age of our cohort.

Colposcopy should be performed in patients with an abnormal PAP smear or abnormal cervix noted at the gynaecological examination; colposcopy is optional in patients with abnormal vaginal discharge or irregular blood loss (10).

Excisional methods (particularly the LLETZ procedure) are considered preferable in situations where invasive cervical 


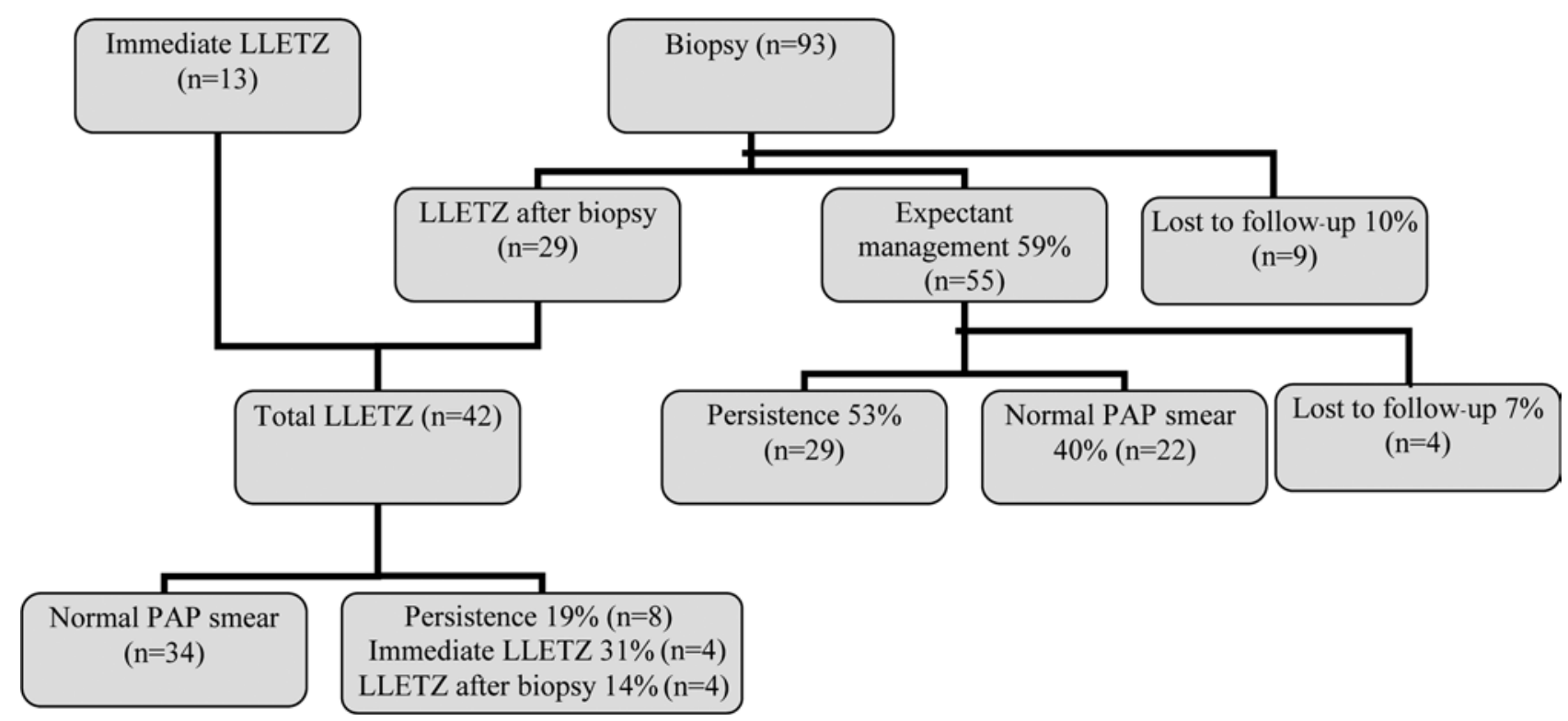

Figure 1. Management of CIN2 lesions. Of the patients who had never had prior cervical interventions, a diagnostic biopsy was performed on 93 patients and a LLETZ procedure was performed on 13 patients. After biopsy, expectant management was selected for 55 patients, and second-visit LLETZ procedures for 29 patients. The rate of persistent disease following expectant management was 53 versus $19 \%$ following treatment with LLETZ.

cancer cannot be precluded via a combination of colposcopy and endocervical sampling with cytological correlation, as well as in situations where the risk of occult cervical cancer is high $(1,10)$. Examples include females with unsatisfactory colposcopic examinations, positive endocervical curettage, and large lesions with a high-grade colposcopic appearance (1). Excisional treatment is mandatory for patients with an unsatisfactory colposcopy, suspicion of invasion or glandular abnormality (11).

According to the guidelines for the management of CIN or adenocarcinoma in situ (AIS), created in 2006 by the American Society for Colposcopy and Cervical Pathology, the options for conservative management in women with CIN2 and CIN3 are extended (1). Observation at colposcopy and cytology at six-month intervals for up to two years is preferred over excisional therapy for reliable adolescents with biopsyconfirmed CIN2 or CIN3, due to the high rate of spontaneous regression and lower rate of progression to invasive carcinoma (6). This is only an option if colposcopy is satisfactory, endocervical curettage is negative, and the possibility of occult disease is acknowledged by the patient $(1,6)$. Repeat biopsy is indicated if high-grade disease persists after 12 months and surgical treatment is indicated if it persists after 24 months (1).

Colposcopy, including a radical excision of the transformation zone at the first visit ('see-and-treat' method), reduces the number of consultations and is thought to reduce patient anxiety while waiting for (repeat) histopathologic results $(12,13)$. In addition, the loss-to-follow-up rate is lower than the $25 \%$ found with the traditional multi-visit management of CIN (14). The analysis revealed that compliance with followup declines over time and reaches significance at the end of 24 months of surveillance (15).

To evaluate the effectiveness of expectant strategy and LLETZ with and without prior biopsy in the management of CIN2 lesions, all data of patients with CIN2 lesions were analysed.
A marginal comment is that the three arms of the study are not completely comparable: i) the immediate LLETZ group was included since CIN2 was found on the histological examination following surgery; ii) no data on the referral history are provided. Conversely, in the group subjected to biopsy, the criteria used to assign the patient to immediate treatment or expectant management depended on the gynaecologist. This does not make the two groups balanced. The expectant management group is more homogeneous. After expectant management, the rate of persistent disease was 53\%. Overall, after diagnostic biopsies no excisional treatment for CIN2 was obtained in $20 \%$ of cases $(n=22)$ due to spontaneous healing. Therefore, any surgical procedure in CIN2 can be considered as overtreatment in almost 1 out of 5 cases.

Immediate radical surgery in patients with PAP3A prevents repeat colposcopic procedures in $12 \%$, but the rate of persistent disease is higher. Following the immediate radical excision of the transformation zone with a LLETZ procedure, significantly more abnormal follow-up smears were observed (31\%) compared to the abnormal follow-up smears noted after a histologic biopsy prior to the LLETZ procedure (14\%).

In the literature, the rate of recurrent or persistent disease is $5-17 \%$ despite therapy with any of the excisional or ablative techniques $(1,14)$. Most failures occur within 2 years of treatment (1). In the present study, the total rate of persistent disease was $7 \%$. Due to the higher rate of persistent disease, radical excisions in the first visit should be restricted. Female individuals who have been treated for CIN2 and CIN3 remain at an increased risk of developing invasive cervical cancer 20 years after treatment for cervical intraepithelial neoplasia (16).

In conclusion, if expectant management in CIN2 is selected following colposcopic and histologic examination, persistent dysplasia is observed in $53 \%$, and $40 \%$ of PAP smears are normal in follow-up. Therefore, any surgical procedure in CIN2 is considered overtreatment in almost 1 
out of 5 of all patients (22/109). Consequently, due to the high rate of persistent disease and the low rate of overtreatment, CIN2 lesions should be treated by excisional procedures. To restrict persistent disease, we recommend a biopsy prior to the actual treatment, since a higher rate of abnormal smears was found in the follow-up after immediate radical excisions in the first visit.

\section{References}

1. Wright TC, Massad LS, Dunton CJ, Spitzer M, Wilkinson EJ and Solomon D: Consensus guidelines for the management of women with cervical intraepithelial neoplasia or adenocarcinoma in situ. American Society for Colposcopy and Cervical Pathology. J Low Genit Tract Disease 11: 223-239, 2007.

2. van Ballegooijen $M$ and Hermens R: Cervical cancer screening in the Netherlands. Eur J Cancer 36: 2244-2246, 2000.

3. De Bruin A, De Koning HJ and Van Ballegooijen $M$ Gezondheidsenquêtes. Cervixuitstrijkjes en mammografieën, Gezondheidsenquêtes 1991 (In Dutch). Maandberichten Gezondheid 5: 4-20, 1993.

4. Werkgroep Oncologische Gynaecologie: Richtlijn cervixcarcinoom, National Guideline The Netherlands 1.2 (In Dutch), 2004.

5. Insinga RP, Glass AG and Rush BB: Diagnoses and outcomes in cervical cancer screening: a population-based study. Am J Obstet Gynaecol 191: 105-113, 2004.

6. Holschneider $\mathrm{CH}$, Goff B and Barss VA: Cervical intraepithelial neoplasia: Management. Up-to-date version 16.1 January 2008.

7. Spitzer M, Apgar BS and Brotzman GL: Management of histologic abnormalities of the cervix. Am Fam Physician 73 105-112, 2006.
8. Östör AG: Natural history of cervical intraepithelial neoplasia: a critical review. Int J Gynaecol Pathol 12: 186-192, 1993.

9. McCredie MRE, Sharples KJ, Paul C, et al: Natural history of cervical neoplasia and risk of invasive cancer in women with cervical intraepithelial neoplasia 3: a retrospective cohort study. Lancet Oncol 9: 425-434, 2008.

10. Werkgroep Oncologische Gynaecologie: Richtlijn cervicale intra-epitheliale neoplasie (CIN), National Guideline The Netherlands 1.1 (In Dutch), 2004.

11. Kyrgiou M, Koliopoulos G, Martin-Hirsch P, Arbyn M, Prendiville W and Paraskevaidis E: Obstetric outcomes after conservative treatment for intraepithelial or early invasive cervical lesions: systematic review and meta-analysis. Lancet 367: 489-498, 2006.

12. Kyrgiou M Tsoumpou I, Vrekoussis T, et al: The up-to-date evidence on colposcopy practice and treatment of cervical intraepithelial neoplasia: the Cochrane colposcopy and cervical cytopathology collaborative group (C5 group) approach. Cancer Treat Rev 32: 516-523, 2006.

13. Dunn TS, Burke M and Shwayder J: A 'see and treat' management for high-grade squamous intraepithelial lesion pap smears. J Low Genit Tract Disease 7:104-106, 2003.

14. Lindeque BG: Management of cervical premalignant lesions. Best Practice \& Research Clinical Obstetrics and Gynaecology 19: 545-561, 2005.

15. Kyrgiou M, Koliopoulos G, Martin-Hirsch P, et al: Management of minor cervical cytological abnormalities: A systematic review and a meta-analysis of the literature. Cancer Treat Rev 33: 514-520, 2007.

16. Kalliala I, Anttila A, Pukkala E and Nieminen P: Risk of cervical and other cancers after treatment of cervical intraepithelial neoplasia: retrospective cohort study. BMJ 331: 1183-1185, 2005. 